\title{
Mikulicz's disease and Küttner's tumor as manifestations of IgG4-related diseases: a review of the literature
}

\author{
Bartłomiej Kamiński ${ }^{1}$, Katarzyna Błochowiak ${ }^{2}$ \\ ${ }^{1}$ Department of Otolaryngology, Maria Skłodowska-Curie District Hospital in Skarżysko-Kamienna, Poland \\ 2Department of Oral Surgery, Poznań University of Medical Sciences, Poland
}

\begin{abstract}
According to a new concept for the classification and division of autoimmune diseases, Mikulicz's disease and Küttner's tumor belong to immunoglobulin G4-related diseases (IgG4-RD) and fulfil their diagnostic criteria. The aim of this study was to summarize the new classification concepts of IgG4-RD in the head and neck area and to review their clinical, histopathological and serologic criteria and the methods used in the diagnostic workup with respect to their advantages, limitations and differentiative value.

The PubMed, Web of Science, Google Scholar, and Scopus databases were searched for articles published between 2009 and 2019 using the following key words: IgG4-related diseases, Mikulicz's disease, Küttner's tumor, salivary glands, xerostomia.

Results of the review of the literature revealed that Mikulicz's disease and Küttner's tumor fulfil the same diagnostic criteria but may manifest different clinical symptoms which determine the choice of the different diagnostic tools.
\end{abstract}

Key words: Mikulicz's disease, Küttner's tumor, IgG4-related diseases.

\section{Introduction}

Immunoglobulin G4 (IgG4)-related diseases (IgG4RD) constitute a group of immune-mediated entities that can affect many organs at nearly any anatomic site. These are chronic, systemic, fibro-inflammatory diseases of unknown etiology. Some allergic, autoimmune and genetic factors can contribute to the disease's development. Autoimmune pancreatitis (AIP) was first described as IgG4-RD, and a relationship between clinical symptoms and increased serum IgG4 was observed. All IgG4$\mathrm{RD}$ are characterized by the formation of infiltrations composed mainly of IgG4+ plasma cells, CD4+ and CD8+ $T$ cells and by fibrosis in the organs that are affected. Depending on the organs involved, these infiltrations lead to organ dysfunction and a special clinical presentation.

Currently, there is a tendency to apply the name IgG4-RD for the same disturbances in different locations and to classify all these disturbances in the same IgG4RD group. Moreover, IgG4-RD may be confused with ma- lignancy, infection or Sjögren's syndrome and vasculitis [1]. These infiltrations usually form pseudotumors and their formation within the head and neck area is one of the most common clinical presentations of IgG4-RD. This area is the second most common site of IgG4-RD presentation after the pancreas [2, 3]. The occurrence of IgG4-RD in the head and neck area is estimated at 20\% [4].

According to the new classification, Mikulicz's disease (MD) and Küttner's tumor (KT) are the main manifestations of IgG4-RD in the salivary glands and are considered as variations of IgG4-related sialadenitis (IgG4-RS). The basic and entry criterion for both MD and KT diagnosis is the involvement of a set of salivary glands. The characteristic involvement generally is defined as an enlargement of the salivary glands or a tumor-like mass within an affected salivary gland. A set of salivary glands refers to both submandibular glands among others. Moreover, the involvement of salivary glands can be determined by either a clinical examination or a radiological examination using a positron 
emission tomography scan or computed tomography. According to the new classification the major salivary gland involvement is bilateral but can be asymmetric [1].

Küttner's tumor is defined as less severe manifestation of the IgG4-RD in the head and neck area with the predominant fibrosis of affected salivary glands and is limited to the required set of the major salivary glands. Küttner's tumor is an enlargement and sclerosing sialadenitis of the submandibular glands (SMG). MD is usually a symmetrical enlargement of the lacrimal glands, parotid and SMG, and sometimes sublingual glands with the symptoms of dry mouth and ocular dryness. However, salivary secretion in MD is normal or decreased to a lesser extent. Xerostomia is not the predominant symptom and the diagnostic criterion. In the past, MD was treated as a subtype of primary Sjögren's syndrome (pSS), but they are now classified as two different diseases. They have different histopathologic, serologic and epidemiologic patterns. In contrast to pSS, the target of autoimmune attack is the parenchyma of the major salivary glands [3]

Furthermore, MD is characterized by non-periductal lymphocytic infiltration with hyperplastic multiple germinal center formation and mild destruction of the acini in contrast to SS [5]. Küttner's tumor is considered as a subtype of MD limited mainly to the SMG and with the predominance of fibrosis, sclerosing sialadenitis and salivary duct destruction.

Both MD and KT fulfil the general criteria for lgG4RD. Diagnosis of IgG4-RD includes the three-step classification process. The first step is based on the clinical or radiological involvement of a typical organ or the presence of a lymphoplasmacytic infiltrate of the same affected organ. The second step consists of all possible clinical, serological, radiological and pathological exclusion criteria. Third step is based on the weighted sum of clinical, serological, radiological and pathological items in inclusion criteria domains. And finally, a case meets the classification criteria for IgG4-RD if the entry criteria are met, no exclusion criteria are present, and the number of total points is $\geq 20$.

These diagnostic criteria were approved in October 2019 by the American College of Rheumatology (ACR) and the European League Against Rheumatism (EULAR) and should be the basis of classifying MD and KT in the IgG4-RD [2, 6]. Due to the location of pathologic lesions in the head and neck region, a dentist may have contact with MD and KT and may correctly diagnose and implement the appropriate therapy. Although both KT and MD belong to the IgG4-RD, they can differ in clinical symptoms, prognosis and disease development. Different diagnostic tools can be useful in KT and MD diagnosis.

The aim of this study was to present and summarize the new classification concepts of IgG4-RD in the sali- vary glands and to review their main clinical, histopathological and serologic criteria and the methods used in the diagnostic workup of IgG4-RD with respect to their advantages, limitations and diagnostic and differentiating value.

\section{Methods}

The authors performed an extensive bibliographic search using PubMed, Web of Science, Google Scholar, and Scopus databases to identify relevant full-text articles in English which were published between January 2009 and October 2019. The following key words were employed in the search of all the selected databases: Mikulicz's disease, Küttner's tumor, salivary glands, xerostomia. We focused on clinical manifestations of IgG4$\mathrm{RD}$ in the head and neck area.

The described research revealed that diagnostics of MD and KT is based on histopathological, radiological and serological tests [2]. However, these criteria can be insufficient for diagnosing IgG4-RS and have different diagnostic usefulness. Radiological examinations of the organs involved cannot efficiently differentiate tumors and IgG4-RD and are of limited value in diagnosing MD and KT. Histopathological and serological findings are more recommended for the final diagnosis of MD and KT and are characterized by typical findings. Clinical manifestation can help in the initial diagnosis and is an indication for salivary gland biopsy in order to obtain glandular specimens for microscopic examination and to measure serum IgG4. These diagnostic steps allow MD and KT to be distinguished from pSS. These entities also have different epidemiological characteristics. IgG4RS, contrary to SS, mainly affects men in middle age [7]. The median age of the onset of IgG4-RD is 58 years. The male-female ratio is approximately $5: 1$ [8]. The characteristic features of IgG4-RD are the slow progression of the disease and a good response to steroid therapy [9].

The main clinical symptoms can develop for many months or even years. High, spiking fever can be reported in the preceding period. These symptoms can mimic infectious diseases. However, according to the new classification criteria both fever and a lack of objective response to glucocorticoids belong to exclusion criteria of IgG4-RD. Recurrent fever is treated as a prominent part of the patient's overall presentation with the underlying disease and as an exclusion criterion of IgG4-RD. No objective response to the initial treatment with prednisone at a minimum of $40 \mathrm{mg}$ daily for a period of 4 weeks is also an exclusion IgG4-RD criterion [1]. Another possible preceding sign in the subclinical period is weight loss [10]. General weakness, malaise and fatigue are rare signs in the initial stage of the disease [11]. Symptoms in 
the preceding period are not characteristic and may also develop in other autoimmune and inflammatory diseases involving the major salivary glands. More helpful information in the diagnostic process of MD comes from the medical history and active period of the disease.

IgG4-RD can affect many organs in the same period or in subsequent stages of the disease. The complications of MD include AIP, retroperitoneal fibrosis, tubulointerstitial nephritis, autoimmune hypophysitis and Riedel's thyroiditis. It is not clear whether these conditions are the direct consequences of MD or other manifestations of IgG4-RD [10]. Furthermore, some specific diseases such as Castleman's disease, Crohn's disease, ulcerative colitis and Hashimoto thyroiditis are defined as exclusion criteria for IgG4-RD [1]. Moreover, 30-40\% of patients with IgG4-RT present allergic entities such as bronchial asthma, chronic rhinitis and eczema. Therefore patients who are suspected of having MD require a comprehensive and thorough examination. Another typical feature of IgG4-RD in the head and neck area is the swelling or enlargement of the major salivary glands. Fibrotic masses in the salivary glands result in pseudotumor formation and should be distinguished from inflammatory and neoplastic processes. Salivary and lacrimal gland involvement in MD generally causes facial and orbital swelling [5]. Mikulicz's disease is characterized by idiopathic, bilateral, symmetrical and painless diffuse swelling of the lacrimal, parotid and SMG $[10,12]$. This swelling lasts for at least 3 months. In MD, gland swelling is persistent, while gland swelling in SS is periodic [13]. Enlargement of the salivary glands in MD is more frequent than in SS. Many autoimmune diseases cause a dysfunction of the exocrine glands, including salivary and lacrimal glands, resulting in dry mouth, xeropthalmia and general dryness of skin [14].

Xerostomia leads to the development of opportunistic infections and oral mucosal manifestations in the oral cavity. It can correspond to selected inflammatory and diagnostic factors of the disease's severity and to histo- pathological changes in salivary glands [15-17]. However, xerostomia is often less severe in IgG4-RD than in SS and it is usually sensitive to immunosuppressive and steroid therapy $[5,18]$. There is a negative correlation between disease duration and the increasing rate of saliva secretion [18]. Decreased salivation in MD and KT is not used in the diagnostic workup of these diseases. Moreover, compared with SS patients, fewer patients with IgG4-RD have symptoms of xerophthalmia or arthralgia $[9,19]$.

Küttner's tumor is considered to be a subtype of MD. In KT, the swelling is well localized and limited mainly to the SMG. In a clinical examination, KT presents with a firm, painless mass in the neck, which mimics the neoplastic process of SMG. Hard and painless salivary gland swelling is the predominant symptom. Küttner's tumor is characterized by asymptomatic bilateral swelling of the SMG. Some patients might experience recurrent pain, discharge, and swelling during eating [20]. The duration of symptoms is highly variable, ranging from less than 1 year to many years [21]. Küttner's tumor could be histopathologically diagnosed by strong lymphocytic infiltration and fibrosis in the SMG either with or without sialolith [22]. Moreover, salivary duct obstruction, salivary stasis, sialolithiasis and secretory dysfunction play a role in the etiology of KT [20]. Sialoliths and mucous plugs are found in $29 \%$ to $83 \%$ of the lesions of KT [23]. Sialoliths found in the course of IgG4-RD are likely to be formed secondarily to sialadenitis and the diminished secretion of saliva [24].

Selected clinicopathologic differences between KT and MD are presented in Table I.

\section{Laboratory diagnostic tests of IgG4-RD}

Although a serum IgG4 concentration > 135 mg/dl has been defined as a general diagnostic criterion of all IgG4-RD, there are no specific antibodies for IgG4RS. Increased serum IgG4 levels give a different number points to the total sum of the diagnostic points. Labora-

Table I. Selected clinicopathologic differences between Mikulicz's disease (MD) and Küttner's tumor (KT)

\begin{tabular}{|c|c|c|}
\hline $\begin{array}{l}\text { Selected clinical, laboratory } \\
\text { and histopathologic features }\end{array}$ & Mikulicz's disease & Küttner's tumor \\
\hline Extra-salivary gland lesion & More frequent & Less frequent, mainly autoimmune pancreatitis \\
\hline Gland swelling & Symmetrical swelling & $\begin{array}{c}\text { Unilateral or bilateral hard swelling, asymmetric } \\
\text { swelling more frequent }\end{array}$ \\
\hline Salivary gland involvement & More than two major salivary glands & Only submandibular glands \\
\hline Lacrimal gland involvement & Often & Rare \\
\hline Xerostomia & Possible but not predominant & Rare \\
\hline $\begin{array}{l}\text { Sialolithiasis, salivary stasis, } \\
\text { mucous plugs }\end{array}$ & Not observed & Observed secondary to sialadenitis \\
\hline Fibrosis & Observed & Predominant \\
\hline
\end{tabular}


tory tests are of additional help in diagnosing IgG4-RS Histopathologic confirmation is the most reliable method to diagnose IgG4-RS. A higher serum IgG4 level is observed in $60-70 \%$ of patients, especially in those with multi-organ involvement [5]. Furthermore, high serum IgG4 concentrations in the absence of histological staining should be used with caution in diagnosing IgG4-RD [25]. Moreover, 30\% of IgG4-RD patients present normal IgG4 levels, and increased serum IgG4 is not exclusively observed in IgG4-RD [26]. IgG4-RD sufferers present higher total IgG, IgG2, IgG4 and IgE serum levels [19].

Although increased serum levels of IgG4 are a diagnostic criterion of IgG4-RD, they can mimic other conditions, such as systemic vasculitides, connective tissue disorders, infections and malignancies. Therefore, measurement of serum lgG4 should be used in screening, not as a diagnostic marker alone [25, 27]. Furthermore, an increased serum IgG4 level correlates with a reduction in saliva production and glandular fibrosis, suggesting that the IgG4 concentration in serum can be a useful marker of the assessment of disease severity and treatment effectiveness. IgM and IgA can also be elevated in IgG4 RD, but not to the same extent as IgG4 [5]. Moreover, high levels of subclasses other than IgG4, e.g. IgG1 and IgG3, can result in hypocomplementemia [3]. Hypocomplementemia is identified in $30 \%$ of IgG4-RD patients [8]. Peripheral blood eosinophilia and increased serum IgE are detected in almost $30 \%$ of patients [5]. However, according to current classification criteria, peripheral eosinophilia, leucopenia, and unexplained thrombocytopenia are exclusion criteria for IgG4-RD diagnosis [1]. In the acute phase of the diseases, the erythrocyte sedimentation rate and C-reactive protein can be elevated to a moderate degree [5].

Some patients have positive low-titer anti-nuclear antibodies and anti-Sjögren's syndrome-related antigen A and B (Ro/SSA) and (La/SSB) and anti-neutrophil cytoplasmic antibodies (ANCA), but their presence can be associated with other immune-mediated diseases. Antinuclear antibodies are detected in 15\% of cases [8]. Moreover, most patients with IgG4-RD are negative for rheumatoid factor (RF) and anti-nuclear antibody (ANA) Other serologic findings such as cryoglobulinemia, positive dsDNA, RPN, Sm antibody, positive SSA/Ro or SSB/ La antibody and other disease-specific autoantibodies can be used in the second step of the diagnostic process to exclude other possible immune-mediated conditions $[2,28,29]$.

\section{Histopathological features}

Histopathological examination of the salivary glands affected remains the gold standard for diagnosing IgG4-RS.
The pathological evidence of an inflammatory process accompanied by a lymphoplasmacytic infiltrate of uncertain etiology in the salivary glands fulfils the entry criterion for MD or KT [1]. Furthermore, there are a few histopathological features defined as exclusion criteria comprising prominent neutrophilic infiltrates, necrotizing vasculitis, markers consistent with inflammatory myofibroblastic tumor, the presence of symptoms of malignancy suggested by cellular atypia, light chain restriction on in situ hybridization studies and multinucleated giant cell formation and granuloma formation suggesting primary granulomatous inflammation [1].

All IgG4-RD present the common histopathological pattern in the affected organs. It includes dense lymphoplasmacytic infiltrates with storiform fibrosis, obliterative phlebitis and predominance of IgG4-bearing plasma cells. However, the severity of fibrosis is dependent on the individual organs involved. Storiform fibrosis and obliterative phlebitis are very seldom found in the salivary glands, contrary to the biliary tract and pancreas [9]. Values of more than 10 lgG4-positive cells per high power field and a ratio of IgG4-positive cells to all IgG-bearing cells > 40-50\% are highly suggestive of IgG4-RD. IgG+ cells can be identified using either lgG staining or CD138 staining [1]. Similar diffuse infiltrations of IgG4-positive plasma cells are found in the patient's submandibular lymph nodes. A ratio of IgG4/lgG-positive cells greater than $40 \%$ meets the diagnostic criteria for IgG4-related lymphadenitis [30].

In contrast to SS, in patients with IgG4-RD, the salivary ducts remain intact. Furthermore, the lower levels of lymphocytic infiltration into the salivary ducts in IgG4-RD may explain the lower rate of dryness, despite the marked swelling of the lachrymal and salivary glands [9]. B-cells form typical germinal centers, whereas T-cells are found in a more scattered pattern. Moreover, most patients with IgG4-RD show polyclonal B-cell proliferation, with equal staining for immunoglobulin $\kappa$ - and $\lambda$-chains [9]. Histologically, it is difficult to distinguish MD from KT. Both KT and MD manifest sialadenitis, as in IgG4-RD. Küttner's tumor is characterized by severe fibrous sclerotic lesions containing lgG4-positive plasma cells. Fibrosis usually tends to be less severe in MD than in KT [9]. Histopathological examination of KT reveals more prominent lymphocytic infiltration, formation of lymphoid follicles, atrophy of glandular parenchyma, advanced hyalinization in the periductal area, and sclerosis. Squamous and goblet cell metaplasia may be detected in the ductal system [20].

On the other hand, fibrosis in MD cannot be examined extensively, because MD is generally diagnosed by LSG biopsy and sclerosis/fibrosis is usually unremarkable in minor salivary gland specimens [9]. Histopathological 
assessment of lesions in KT may evolve through four histological stages. In the initial stage, focal chronic inflammation with nests of lymphocytes in the periductal area predominates. Salivary ducts are moderately dilated and contain inspissated secretions. In more advanced stages, lymphocytic infiltrations become diffuse with severe periductal fibrosis. Fibrosis is present in the centers of the lobules, accompanied by atrophy of the acini. Most advanced stages of KT present cirrhosis-like stroma with marked parenchymal loss and sclerosis [21, 31-34].

\section{Radiographic examination in IgG4-RS}

There are only a few potentially useful imaging modalities in IgG4-RD diagnosis. They can be used for both exclusion and inclusion criteria. Well-known radiological findings suspicious for malignancy or infection such as rapid radiological progression of the lesions, splenomegaly and long bone abnormalities consistent with Erdheim-Chester disease are exclusion criteria of IgG4-RD [1]. In the head and neck area rapid progression of radiological symptoms can be easily observed and is defined as significant worsening within a 4-6 week interval [1].

Scintigraphy reveals significant accumulation of ${ }^{67} \mathrm{Ga}$ in salivary glands affected by MD. 2-[18 F]-fluoro-2-deoxy-D-glucose positron emission tomography/computed tomography (FDG-PET/CT) demonstrates abnormal ${ }^{18} \mathrm{~F}-\mathrm{FDG}$ uptake in salivary glands involved with IgG4-RD. Ultrasonic examinations of the glands involved might be efficient for screening IgG4-DS, and are also useful for the follow-up of this disease after corticosteroid therapy. Ultrasonic assessment of the salivary glands in IgG4RD includes the detection of hypoechoic areas, hyperechoic lines or spots, and reticular and nodal patterns in the parotid glands [13].

Unfortunately, multiple hypoechoic areas and hyperechoic lines or spots in the parotid glands can be detected in both IgG4-RD and SS. These findings might be misleading. However, hypoechoic areas of IgG4-DS are observed in the normal parotid parenchyma without a reduction in the echo intensity level and heterogeneity. Moreover, IgG4-DS mainly affects the SMG, and most of the parotid glands present normal echogenicity [13]. In SS patients, atrophic changes are found in both the parotid and SMG [13]. Ultrasonic examination in KT reveals diffuse changes in the SMG in the form of multiple hypoechoic lesions, heterogeneous background and duct dilatation $[20,35]$. Therefore, for the ultrasonic diagnosis of MD and KT a comprehensive assessment of both submandibular and parotid salivary glands is needed. To detect focal salivary masses in KT, ultrasonography has a sensitivity of $100 \%$ and an accuracy of nearly $100 \%$ compared with $92 \%$ and $87 \%$ by palpation [21].
A more useful tool for differentiating IgG4-RS from SS is Doppler ultrasonography. The nodal and reticular patterns of IgG4-DS show high vascularity, while SS exhibits small dot-like vascularity in the parotid glands. Moreover, sonography is helpful in diagnosing salivary gland swelling and assessing adjacent lymph nodes. Nodal or reticular changes on both sides of the swollen salivary gland allow tumor lesions to be excluded.

Computed tomography (CT) and magnetic resonance imaging (MRI) detect an enlargement of the salivary glands with nonspecific findings, because most patients with enlarged salivary glands can be misdiagnosed as having IgG4-DS [13]. Sialography is not recommended for diagnosing IgG4-RS, because there is no punctate or globular sialectasis on sialograms in IgG4-DS, while it is generally observed in SS [13].

The most important issue in diagnosing IgG4-DS is the differentiation of malignant lymphoma. Malignant lymphoma sometimes affects the bilateral salivary glands, which mimics IgG4-DS. To make a final diagnosis, a biopsy is recommended. IgG4-DS does not always occur simultaneously in both the lacrimal and salivary glands and radiographic examination can be repeated and used for the final diagnosis and for disease monitoring. There are no definitive radiological features of $\mathrm{MD}$ and $\mathrm{KT}$ but the role of radiological findings in comprehensive IgG4-RD diagnosis is increasing. They are useful in the second step of IgG4-RD diagnosis to exclude the possible malignancy or infectious disease of the involved organs. Moreover, radiological findings in isolation without positive clinical, serological or pathological data are not sufficient for clinical diagnosis or for final disease confirmation [1].

\section{Salivary gland biopsy in diagnosing IgG4-RS}

According to the 2019 ACR/EULAR IgG4-RD classification criteria, the impact of biopsy in the diagnostic process was limited. Although biopsies are essential in many settings to establish the diagnosis of IgG4-RD and to exclude other infectious diseases or malignancies of the involved salivary glands, they are not required when the diagnosis of MD and KT is based on clinical, serological and radiological findings. Currently, there is a tendency to introduce only the serological, clinical and radiological criteria for IgG4-RD diagnosis and to avoid more invasive surgical approaches. The excisional biopsies are more recommended in the treatment than in the diagnostic process. They are limited to the most severe clinical manifestations of MD or KT. The pathological assessment of the involved salivary glands can be made routinely using surgical resection specimens. They demonstrate the full range of findings considered 
characteristic of IgG-RD. Although excisional biopsies in the form of submandibulectomy or parotidectomy provide the most reliable diagnostic specimens, the MD and KT diagnosis can be made using small biopsy samples that frequently do not demonstrate the full spectrum of pathological findings.

Another tendency is to introduce less invasive surgical approaches for diagnosing lgG4-RD. Moreover, 9\% of the IgG4-RD cases were diagnosed without a biopsy and $37 \%$ of patients with IgG4-RD did not have classic histopathological findings and did not meet previously defined histopathological criteria [36].

There are a few surgical approaches to obtain salivary gland specimens for microscopic evaluation. Labial salivary gland (LSG) biopsy and incisional biopsy of the submandibular glands (SMG) indicate similar sensitivity, but SMG is more recommended for MD detection. Furthermore, submandibulectomy and submandibular lymph node biopsy are alternative options in KT. In the diagnosis of sialadenitis, a salivary gland biopsy is recommended. The results of labial salivary gland (LSG) biopsy are included in the comprehensive diagnostic process of SS. LSG biopsy has diagnostic value in sarcoidosis, lymphoma, non-specific sialadenitis and amyloidosis [37]. Incisional biopsy of the SMG is useful for diagnosing IgG4-DS, in addition to being a less invasive procedure than excisional biopsy under general anesthesia. On the other hand, LSG biopsy may be less suitable as a single procedure because of its low sensitivity and poor correlation with the histology of the SMG. The sensitivity, specificity and accuracy of SMG biopsies have been reported as being 100\%. The same parameters in LSG biopsies have been reported at 69.2\%, 100\% and $71.4 \%$, respectively [5].

In cases of KT presenting with severe fibrosis of the SMG, a submandibulectomy is performed for definitive diagnosis and even for treatment. However, this invasive procedure often results in postoperative complications, including bleeding, facial nerve palsy, amblygeustia and hyposalivation. Therefore, the incisional biopsy of the SMG and LSG biopsy are less invasive procedures than submandibulectomy and are recommended for the diagnosis of IgG4-RS [5]. Both incisional SMG and LSG biopsies can be performed under local anesthesia. Furthermore, swelling in the SMG is the main recommendation for incisional biopsy. SMG biopsy in IgG4-RD specimens allows the detection of strong lymphocytic infiltration with hyperplastic germinal centers, mild destruction of the acini, and selective infiltration by lgG4-positive plasma cells with an IgG4-positive plasma cells/lgG-positive plasma cells ratio $>0.4$.

In contrast, although LSG specimens can show similar histological findings, specimens usually present only mild lymphocytic infiltration with an IgG4-positive plasma cells/lgG-positive plasma cells ratio $\leq 0.4$ and without germinal centers. Minor salivary gland biopsy such as LSG biopsy may sometimes be substituted when biopsy of the major salivary glands is difficult. The sensitivity of detection of IgG4+ plasma cells in LSG specimens is relatively low [38]. In addition to glandular tissue, submandibular lymph nodes specimens can be easily obtained for microscopic examination [30]. The presence of lymphocytic infiltration in the lymph nodes and a ratio of lgG4/lgG greater than $40 \%$ are the histopathological criterion of IgG4-related lymphadenitis [30].

Another possible option in diagnosing KT is fine needle aspiration biopsy [39]. Although specimens of KT contain relatively large numbers of lymphoid cells and should be differentiated from malignant lymphoma of the SMG, some cytopathological features of KT may result in misinterpretation. Therefore, it is not a reliable method for diagnosing KT [20, 37]. The utility of the parotid gland in diagnosing IgG4-RD has not been reported so far. Parotid biopsy can be recommended in patients who have a negative minor salivary gland biopsy result.

Comparative studies suggest that both procedures - sublingual and parotid biopsy - retain diagnostic potential comparable to that of lip biopsy and may be associated with lower postoperative morbidity. There are a few alternative types of salivary gland biopsy in MD diagnosis, whereas SMG biopsy remains the most reliable test for KT [40].

\section{Conclusions}

The new concept for the classification and division of MD and KT requires a new multidisciplinary and comprehensive approach to their diagnosis and therapy. Specific serologic and histopathologic changes modified by the immunological system reflect their multifactorial etiology and systemic characteristics, even in the case of most localized organ involvement, such as sialadenitis related to $\mathrm{MD}$ and $\mathrm{KT}$.

The authors declare no conflict of interest.

\section{References}

1. Wallace ZS, Naden RP, Chari S, et al. Members of the ACR/EULAR IgG4-RD Classification Criteria Working Group. The 2019 American College of Rheumatology/European League Against Rheumatism Classification Criteria for IgG4-related Disease. Ann Rheum Dis 2020; 79: 77-87, DOI: 10.1136/annrheumdis-2019-216561.

2. Sebastian A, Dionizy P, Wiland P. IgG4-Related Disease and the Spectrum of Mimics in Rheumatology. In: Chronic autoimmune epithelitis. Sjögren's syndrome and other autoim- 
mune diseases of the exocrine glands, Maslinska $M$ (ed.) IntechOpen Book Series Rheumatology, Vol. 3. London 2019 73-85, DOI: 10.5772/intechopen.73892.

3. Gallo A, Martellucci S, Fusconi M, et al. Sialendoscopic management of autoimmune sialadenitis: a review of literature. Acta Otorhinolaryngol Ital 2017; 37: 148-154, DOI: 10.14639/0392-100X-1605.

4. Ochoa ML, López BG, Cabello RR, Feregrino RR. IgG4-related multiorgan disease: report of the first autopsy case. BMJ Case Rep 2013; 2013: bcr2013009636, DOI: 10.1136/bcr-2013009636.

5. Moriyama M, Furukawa S, Kawano S, et al. The diagnostic utility of biopsies from the submandibular and labial salivary glands in IgG4-related dacryoadenitis and sialoadenitis, socalled Mikulicz's disease. Int J Oral Maxillofac Surg 2014; 43: 1276-1281, DOI: 10.1016/j.ijom.2014.06.014.

6. Della-Torre E, Lanzillotta $M$, Doglioni C. Immunology of IgG4-related disease. Clin Exp Immunol 2015; 181: 191-206.

7. Tanaka K, Harada H, Kayamori K, Omura K. Chronic Sclerosing Sialadenitis of the Submandibular Gland as the Initial Symptom of IgG4-Related Disease: A Case Report. Tohoku J Exp Med 2015; 236: 193-198, DOI: 10.1620/tjem.236.193.

8. Rolla D, Bellino D, Peloso G, et al. The first case of IgG4related disease in Italy. J Nephropathol 2013; 2: 144-149, DOI: 10.12860/JNP.2013.24.

9. Umehara H, Okazaki K, Masaki Y, et al. A novel clinical entity, IgG4-related disease (IgG4RD): general concept and details. Mod Rheumatol 2012; 22: 1-14, DOI: 10.1007/s10165-011-0508-6.

10. Wu Y, Xu ZR, Zhou WJ, Yang YM. Immunoglobulin G4-related disease with features of Mikulicz's disease and autoimmune pancreatitis which firstly presented as asymptomatic lymphadenopathy: a case report. Chin Med J (Engl) 2015; 128: 706 707, DOI: 10.4103/0366-6999.151702.

11. Bhagwat NM, Tayde PS, Dalwadi PP, et al. Mikulicz's Disease with hypophysitis - a new IgG4-mediated disorder. Endokrynol Pol 2016; 67: 622-626, DOI: 10.5603/EP.2016.0071.

12. Takano K, Yamamoto M, Takahashi H, et al. Clinicopatholog ic similarities between Mikulicz disease and Küttner tumor Am J Otolaryngol 2010; 31: 429-434, DOI: 10.1016/j.amjoto.2009.08.002.

13. Shimizu M, Okamura K, Kise Y, et al. Effectiveness of imaging modalities for screening lgG4-related dacryoadenitis and sialadenitis (Mikulicz's disease) and for differentiating it from Sjögren's syndrome (SS), with an emphasis on sonography. Arthritis Res Ther 2015; 17: 223, DOI: 10.1186/s13075-0150751-x.

14. Błochowiak K, Olewicz-Gawlik A, Polańska A, et al. Oral mucosal manifestations in primary and secondary Sjögren's syndrome and dry mouth syndrome. Postepy Dermatol Alergol 2016; 33: 23-27, DOI: 10.5114/pdia.2016.57764.

15. Błochowiak KJ, Trzybulska D, Olewicz-Gawlik A, et al. Levels of EGF and VEGF in patients with primary and secondary Sjögren's syndrome. Adv Clin Exp Med 2018; 27: 455-461, DOI: 10.17219/acem/70800.

16. Błochowiak KJ, Olewicz-Gawlik A, Trzybulska D, et al. Serum ICAM-1, VCAM-1 and E-selectin levels in patients with primary and secondary Sjögren's syndrome. Adv Clin Exp Med 2017; 26: 835-842, DOI: 10.17219/acem/61434
17. Kamiński B, Guzera Z. The unknown face of labial salivary glands. Labial salivary gland biopsy as a diagnostic tools. Medical Studies/Studia Medyczne 2019; 35: 172-179, DOI: 10.5114/ ms.2019.86337.

18. Moriyama M, Tanaka A, Maehara T, et al. Clinical characteristics of Mikulicz's disease as an IgG4-related disease. Clin Oral Investig 2013; 17: 1995-2002, DOI: 10.1007/s00784-0120905-z.

19. Masaki Y, Dong L, Kurose N, et al. Proposal for a new clinical entity, IgG4-positive multiorgan lymphoproliferative syndrome: analysis of 64 cases of IgG4-related disorders. Ann Rheum Dis 2009; 68: 1310-1315, DOI: 10.1136/ard.2008.089169.

20. Poghosyan A, Misakyan M, Sargsyan A, et al. Chronic sclerosing sialadenitis (Küttner's tumor) of the submandibular salivary gland: Our experience of one case report. Clin Case Rep 2019; 7: 1600-1604, DOI: 10.1002/ccr3.2303.

21. Uhliarova B, Svec M. Kuttner tumor. Bratisl Lek Listy 2013; 114: 36-38, DOI: 10.4149/bll 2013009.

22. Furukawa S, Moriyama M, Kawano S, et al. Clinical relevance of Küttner tumour and IgG4-related dacryoadenitis and sialoadenitis. Oral Dis 2015; 21: 257-262, DOI: 10.1111/odi.12259.

23. Beriat GK, Akmansu SH, Kocatürk S, Ataoğlu O. Chronic Sclerosing Sialadenitis (Küttner's tumour) of the Parotid Gland. Malays J Med Sci 2010; 17: 57-61.

24. Gontarz M, Wyszyńska-Pawelec G, Zapała J, et al. IgG4-related disease in the head and neck region: report of two cases and review of the literature. Pol J Pathol 2016; 67: 370-375, DOI: 10.5114/pjp.2016.65871.

25. Beyer G, Schwaiger T, Lerch MM, Mayerle J. IgG4-related disease: a new kid on the block or an old acquaintance? United European Gastroenterol J 2014; 2: 165-172, DOI: $10.1177 / 2050640614532457$.

26. Deng C, Li W, Chen S, et al. Histopathological diagnostic value of the IgG4+/lgG+ ratio of plasmacytic infiltration for IgG4-related diseases: a PRISMA-compliant systematic review and meta-analysis. Medicine (Baltimore) 2015; 94: e579, DOI: 10.1097/MD.0000000000000579.

27. Yun J, Wienholt L, Adelstein S. Poor positive predictive value of serum immunoglobulin G4 concentrations in the diagnosis of immunoglobulin G4-related sclerosing disease. Asia Pac Allergy 2014; 4: 172-176, DOI: 10.5415/apallergy.2014.4.3.172.

28. Carruthers MN, Khosroshahi A, Augustin T, et al. The diagnostic utility of serum IgG4 concentrations in IgG4-related disease. Ann Rheum Dis 2015; 74: 14-18, DOI: 10.1136/annrheumdis-2013-204907.

29. Hao M, Liu M, Fan G, et al. Diagnostic value of serum IgG4 for IgG4-related disease: a PRISMA-compliant systematic review and meta-analysis. Medicine (Baltimore) 2016; 95: e3785, DOI: 10.1097/MD.0000000000003785.

30. Qu LM, Liu YH, Brigstock DR, et al. IgG4-related autoimmune pancreatitis overlapping with Mikulicz's disease and lymphadenitis: a case report. World J Gastroenterol 2013; 19: 94909494, DOI: 10.3748/wjg.v19.i48.9490.

31. Sun L, Zhou Q, Brigstock DR, et al. Focal autoimmune pancreatitis and chronic sclerosing sialadenitis mimicking pancreatic cancer and neck metastasis. World J Gastroenterol 2014; 20: 17674-17679, DOI: 10.3748/wjg.v20.i46.17674. 
32. Mochizuki Y, Omura K, Kayamori K, et al. Küttner's tumor of the sub-mandibular gland associated with fibrosclerosis and follicular hyperplasia of regional lymph nodes: a case report. J Med Case Rep 2011; 5: 121.

33. Putra J, Ornstein DL. Küttner Tumor: IgG4-Related Disease of the Submandibular Gland. Head Neck Pathol 2016; 10: 530 532, DOI: 10.1007/s12105-016-0729-2.

34. Geyer JT, Ferry JA, Harris NL, et al. Chronic Sclerosing Sialadenitis (Küttner Tumor) Is an IgG4-associated Disease. Am J Surg Pathol 2010; 34: 202-210, DOI: 10.1097/PAS.0b013e3181c811ad.

35. Shimizu M, Moriyama M, Okamura K, et al. Sonographic diagnosis for Mikulicz disease. Oral Surg Oral Med Oral Patho Oral Radiol Endodont 2009; 108: 105-113, DOI: 10.1016/j. tripleo.2009.02.032.

36. Baer AN, Gourin CG, Westra WH, et al. Sjögren's International Collaborative Alliance. Rare diagnosis of IgG4-related systemic disease by lip biopsy in an international Sjögren syndrome registry. Oral Surg Oral Med Oral Pathol Oral Radiol 2013; 115: e34-39, DOI: 10.1016/j.00oo.2012.07.485.

37. Deshpande V, Zen Y, Chan JKC, et al. Consensus statement on the pathology of IgG4-related disease. Mod Pathol 2012; 25 : 1181-1192, DOI: 10.1038/modpathol.2012.72.

38. Masaki Y, Kurose N, Umehara H. IgG4-related disease: a novel lymphoproliferative disorder discovered and established in Japan in the 21st century. J Clin Exp Hematop 2011; 51: 13-20, DOI: 10.3960/jslrt.51.13.

39. Leon ME, Santosh N, Agarwal A, et al. Diagnostic Challenges in the Fine Needle Aspiration Biopsy of Chronic Sclerosing Sialadenitis (Küttner's Tumor) in the Context of Head and Neck Malignancy: A Series of 4 Cases. Head Neck Pathol 2016; 10: 389-393, DOI: 10.1007/s12105-016-0701-1.

40. Błochowiak K, Sokalski J. Labial salivary gland biopsy in the diagnosis of Sjögren's syndrome. Eur J Clin Exp Med 2019; 17: 162-168, DOI: 10.15584/ejcem.2019.2.10. 